\title{
Polymorphic duplicate genes and persistent non-coding sequences reveal heterogeneous patterns of mitochondrial DNA loss in salamanders
}

Rebecca A. Chong ${ }^{1,2^{*}}$ and Rachel Lockridge Mueller ${ }^{1}$

\begin{abstract}
Background: Mitochondria are the site of the citric acid cycle and oxidative phosphorylation (OXPHOS). In metazoans, the mitochondrial genome is a small, circular molecule averaging $16.5 \mathrm{~kb}$ in length. Despite evolutionarily conserved gene content, metazoan mitochondrial genomes show a diversity of gene orders most commonly explained by the duplication-random loss (DRL) model. In the DRL model, (1) a sequence of genes is duplicated in tandem, (2) one paralog sustains a loss-of-function mutation, resulting in selection to retain the other copy, and (3) the non-functional paralog is eventually deleted from the genome. Despite its apparent role in generating mitochondrial gene order diversity, little is known about the tempo and mode of random gene loss after duplication events. Here, we determine mitochondrial gene order across the salamander genus Aneides, which was previously shown to include at least two DRL-mediated rearrangement events. We then analyze these gene orders in a phylogenetic context to reveal patterns of DNA loss after mitochondrial gene duplication.
\end{abstract}

Results: We identified two separate duplication events that resulted in mitochondrial gene rearrangements in Aneides; one occurred at the base of the clade tens of millions of years ago, while the other occurred much more recently (i.e. within a single species), resulting in gene order polymorphism and paralogs that are readily identifiable. We demonstrate that near-complete removal of duplicate rRNA genes has occurred since the recent duplication event, whereas duplicate protein-coding genes persist as pseudogenes and duplicate tRNAs persist as functionally intact paralogs. In addition, we show that non-coding DNA duplicated at the base of the clade has persisted across species for tens of millions of years.

Conclusions: The evolutionary history of the mitochondrial genome, from its inception as a bacterial endosymbiont, includes massive genomic reduction. Consistent with this overall trend, selection for efficiency of mitochondrial replication and transcription has been hypothesized to favor elimination of extra sequence. Our results, however, suggest that there may be no strong disadvantage to extraneous sequences in salamander mitochondrial genomes, although duplicate rRNA genes may be deleterious.

Keywords: Duplication-random loss model, Gene rearrangement, Pseudogene, Mitochondrial genome evolution, Repetitive DNA, Aneides

\footnotetext{
* Correspondence: rachong@hawaii.edu

1Department of Biology, Colorado State University, Fort Collins, CO

80523-1878, USA

2Present address: Department of Biology, University of Hawai'i at Mānoa,

Honolulu, HI 96822, USA
} 


\section{Background}

Mitochondria are the site for the citric acid cycle and oxidative phosphorylation (OXPHOS), the final steps of ATP synthesis via cellular respiration. These intracellular organelles have their own genome, which in metazoans is a small, circular molecule averaging $16.5 \mathrm{~kb}$ in length $[1,2]$. Metazoan mitochondrial genomes typically encode 13 electron transport proteins, two rRNAs, and 22 tRNAs [2]. This gene content is evolutionarily conserved, likely reflecting the fitness advantage of having the proteins involved in primary electron transfer (i.e. redox chemistry) co-localized with the genes that encode them, allowing direct redox control of gene expression [3]. Other hypotheses to explain why this conserved set of genes has remained in a distinct mitochondrial genome, rather than being transferred to the nuclear genome, include (1) their role in redox damage sensing [4], and (2) the hydrophobicity of their protein products, which precludes their import back into the mitochondria [5]. In contrast to such evolutionarily conserved gene content, metazoan mitochondrial genomes show a diversity of gene orders that result from gene rearrangement [1, 6-8]. Many mitochondrial gene orders are hypothesized to reflect rearrangement by neutral processes $[9,10]$, although tRNA positions within the vertebrate mitochondrial genome have been hypothesized to reflect selection for translational capacity [11].

The most commonly invoked model for mitochondrial gene rearrangement is the duplication-random loss (DRL) model. In the DRL model, a sequence of genes is initially duplicated in tandem. Following this initial duplication, one paralog sustains a loss-of-function mutation, resulting in strong selective pressure to retain the other gene copy functionally intact. The non-functional paralog continues to accumulate mutations and is eventually removed from the genome by deletion. Depending on which gene copy is ultimately deleted, this process may restore the original gene order or result in a novel gene order $[12,13]$.

The DRL model has been invoked to explain the variation in mitochondrial gene order observed in diverse animal clades including lizards, insects, and caecilians [8, 14-18]. Evidence for the DRL model includes the presence of mitochondrial pseudogenes that can be identified as paralogs of rearranged genes [19]. Despite its apparent role in generating mitochondrial gene order diversity, little is known about the tempo and mode of random gene loss after duplication events. For example, does loss of gene duplicates occur slowly, reflecting rare deletion events and/or their fixation solely by genetic drift? Alternatively, does loss of gene duplicates occur quickly, reflecting frequent deletion events and/or positive selection for deletions?

Selection would play a role in the deletion of duplicate genes if their presence in the genome negatively impacted mitochondrial function. This could occur by increasing the time required to complete transcription of mitochondrial genes and/or replication of the mitochondrial genome $[20,21]$. It could also occur by increasing susceptibility to harmful gain-of-function mutations [22] or by shifting genes to a more vulnerable position along the mutation gradient [23]. In such cases, selection would favor the elimination of all extraneous sequence, irrespective of its identity. Alternatively, expression of mitochondrial pseudogenes could result in the production of non-functional proteins [24]. Finally, expression of additional functional gene copies could result in changes to the stoichiometry of mitochondrial proteins $[25,26]$. In these latter two cases, selection would favor mutations that delete these specific sequences or otherwise diminish their contribution to the mitochondrial proteome.

Much of our current understanding of mitochondrial gene order variation comes from comparisons across highly divergent clades such as bird families and amphibian orders, where rearrangements have been fixed deep in evolutionary time $[1,19,27,28]$. In these systems, gene loss occurred far enough in the past that little or no trace of duplicated sequences remain in the genomes of extant taxa; this precludes the study of the tempo and mode of paralog loss. In contrast, the study of more recent gene rearrangements - where duplicates and intermediate stages of loss are polymorphic within species or fixed between closely related lineages - allow the study of random loss "in action." Such studies remain relatively rare $[29,30]$.

Salamanders, an amphibian clade of 708 species [31], include both typical vertebrate mitochondrial genomes and a number of independently derived gene rearrangements consistent with origination through the DRL model [19]. Because this mitochondrial genomic diversity is unusual among vertebrates, salamanders are a promising taxon for identifying recent gene rearrangements appropriate for studies of tempo and mode of duplicate gene loss. Within salamanders, the six-species genus Aneides includes two species - A. hardii and A. flavipunctatus - that have distinct, derived mitochondrial gene orders resulting from gene duplications $[19,32]$. The duplication in A. flavipunctatus encompassed protein coding genes ND6 and cytb, tRNA genes $\mathrm{E}, \mathrm{T}$, and $\mathrm{P}$, and an intergenic spacer found in diverse salamander clades [19, 28, 33, 34]. The mitochondrial genome of $A$. hardii shows evidence of having undergone two duplication events. The first duplication involves a nearly identical region of the genome duplicated in A. flavipunctatus, suggesting that it may have occurred in the common ancestor of these two species. The second duplication in $A$. hardii involves part of the first duplication as well as the control region, 12S, 16S, ND1, and tRNAs F, V, and L [19].

For this study, we examined mitochondrial gene order in multiple population-level lineages of Aneides in a 
phylogenetic context to pinpoint the evolutionary origins of gene duplications. Our goals were to (1) determine whether the similar duplications in A. hardii and A. flavipunctatus are, in fact, a synapomorphy for some/all species in the genus, (2) determine whether variation in gene order exists between any closely related lineages or within species, and (3) examine the tempo and mode of duplicate gene loss. We identified two separate duplication events that resulted in mitochondrial gene rearrangements. One such rearrangement occurred at the base of the clade tens of millions of years ago, while the other occurred much more recently (i.e. within a single species), resulting in gene order polymorphism and paralogs that are readily identifiable. We demonstrate that near-complete removal of duplicate rRNA genes has occurred since the recent duplication event, whereas duplicate protein-coding genes persist as pseudogenes and duplicate tRNAs persist as functionally intact paralogs. In addition, we show that non-coding DNA duplicated at the base of the clade has persisted across species for tens of millions of years. Taken together, our results suggest that, although there may be no strong disadvantage to extraneous sequences in salamander mitochondrial genomes, duplicate rRNA genes may be deleterious.

\section{Methods}

\section{Taxon selection and sampling strategy}

Our first objective was to estimate a comprehensive phylogeny for Aneides to provide the evolutionary context for analysis of mitochondrial gene rearrangements. To this end, we collected nuclear gene sequence data from four individuals for all six species in the clade (Table 1). We included additional samples of A. flavipunctatus because previous studies have documented high levels of divergence across populations in this species [35-37]. We also included an additional sample of $A$. hardii because a previous study identified a novel gene order in this species [19]. We included four related plethodontid salamander species as outgroups (Ensatina eschscholtzii, Hydromantes brunus, Plethodon elongatus, and Desmognathus fuscus) [32, 38, 39]. All work was completed in compliance with Colorado State University's Institutional Animal Care and Use Committee (Protocol 08-184A-02).

Our second objective was to compare gene order both within and among species of Aneides to pinpoint the evolutionary origins of the gene duplications underlying gene rearrangement. To this end, we collected mitochondrial genome sequence data from individuals that represent nine divergent population-level lineages of Aneides (Table 2). Wherever possible, we collected mitochondrial genome sequence data from individuals sampled in the phylogenetic analysis to maximize overlap between the two datasets. As above, we sampled multiple individuals from both A. flavipunctatus and A. hardii.

\section{DNA amplification and sequencing for phylogenetic analyses}

For each sample, we extracted total genomic DNA from either flash-frozen or RNAlater-preserved (Qiagen) liver or tail tissue using the Puregene kit (Qiagen) according to the manufacturer's protocols. We amplified a portion of the nuclear-encoded genes BDNF, POMC, and RAG1 using the Polymerase Chain Reaction (PCR), which produced amplicons of 720 bps, 489 bps, and 837 bps for each gene, respectively. Primers and PCR reaction conditions were obtained from previously published studies [38]. Briefly, approximately $10 \mathrm{ng}$ of genomic DNA was amplified using $0.25 \mathrm{mM}$ dNTPs, $0.5 \mu \mathrm{M}$ of each primer, $0.5 \mathrm{U} \mathrm{Taq}, 25 \mu \mathrm{M} \mathrm{MgCl}$, and $10 \times$ reaction buffer (no $\mathrm{MgCl}_{2}$ ) in a $12.5 \mu \mathrm{L}$ reaction volume. Genes were PCRamplified for 35 cycles $\left(95{ }^{\circ} \mathrm{C} 45 \mathrm{~s}, 56-58{ }^{\circ} \mathrm{C} 1 \mathrm{~min}\right.$, and $\left.72{ }^{\circ} \mathrm{C} 1 \mathrm{~min}\right)$. All PCR products were electrophoresed on a $1 \%$ agarose SB gel stained with Gel Red (Biotium Inc.) and visualized under ultraviolet light. PCR products were purified using ExoSAP-IT (Affymetrix USB products). PCR products were sequenced in both directions by the University of Chicago Comprehensive Cancer Center's DNA sequencing and genotyping facility using direct double-strand cycle sequencing with Big Dye v3.1 chemistry (Perkin-Elmer) and an ABI 3730 automated sequencer. Contiguous DNA sequences were aligned and edited using Geneious version R7 (Biomatters), and multiple sequence alignments were initially generated using Muscle v.3.6 [40] and subsequently verified by eye. The open reading frames were also verified for all genes using Geneious R7. All sequences are deposited in GenBank (accession numbers MF946473-MF946547).

\section{Phylogenetic analyses}

We estimated phylogenetic relationships within the genus Aneides using both Maximum Likelihood (ML) and Bayesian Inference (BI) on the aligned, concatenated nuclear-gene dataset (BDNF, POMC, and RAG1). We evaluated multiple data partitioning strategies in order to incorporate evolutionary information specific to each gene and codon position. We determined three appropriate data partitions using PartitionFinder v.1.1.1 [41]: (1) codon positions 1 and 2 for BDNF, (2) codon positions 1 and 2 for POMC and RAG1 combined, and (3) codon position 3 for all three genes combined. Using the Akaike Information Criterion to select the best model of nucleotide substitution, we determined JC, F81 + I, and GTR $+\Gamma$ for each partition, respectively.

We conducted partitioned Bayesian analyses in MrBayes v.3.2 [42] for the concatenated nuclear-gene dataset. We conducted two independent searches consisting of three 
Table 1 Specimen information for individuals included in this study and their voucher numbers, locality information, and GenBank accession numbers for nuclear loci

\begin{tabular}{|c|c|c|c|c|c|c|}
\hline Species & Voucher & State: County & BDNF & POMC & RAG1 & Source \\
\hline Aneides aeneus & $\mathrm{DH} 74978$ & GA: Chatooga & MF946473 & MF946498 & MF946523 & This study \\
\hline Aneides aeneus & DH74985 & GA: Chatooga & MF946474 & MF946499 & MF946524 & This study \\
\hline Aneides aeneus & DH77583 & KY: Letcher & MF946475 & MF946500 & MF946525 & This study \\
\hline Aneides aeneus & DH77584 & KY: Letcher & MF946476 & MF946501 & MF946526 & This study \\
\hline Aneides ferreus & MVZ219942 & CA: Siskiyou & MF946477 & MF946502 & MF946527 & This study \\
\hline Aneides ferreus & MVZ219953 & OR: Douglas & MF946478 & MF946503 & MF946528 & This study \\
\hline Aneides ferreus & MVZ219958 & OR: Linn & MF946479 & MF946504 & MF946529 & This study \\
\hline Aneides ferreus & RCT545 & CA: Del Norte & MF946480 & MF946505 & MF946530 & This study \\
\hline Aneides flavipunctatus & AGC299 & CA: Santa Cruz & MF946481 & MF946506 & MF946531 & This study \\
\hline Aneides flavipunctatus & MVZ219973 & CA: Siskiyou & EU275895 & EU275849 & EU275809 & Vieites et al. [38] \\
\hline Aneides flavipunctatus & MVZ219977 & CA: Sonoma & MF946482 & MF946507 & MF946532 & This study \\
\hline Aneides flavipunctatus & RAC080 & CA: Mendocino & MF946483 & MF946508 & MF946533 & This study \\
\hline Aneides flavipunctatus & RCT481 & CA: Shasta & MF946484 & MF946509 & MF946534 & This study \\
\hline Aneides flavipunctatus & RLM172 & CA: Del Norte & MF946485 & MF946510 & MF946535 & This study \\
\hline Aneides hardii & MVZ226110 & NM: Otero & EU275857 & EU275811 & EU275780 & Vieites et al. [38] \\
\hline Aneides hardii & RAC020 & NM: Lin & MF946486 & MF946511 & MF946536 & This study \\
\hline Aneides hardii & RAC025 & NM: Otero & MF946487 & MF946512 & MF946537 & This study \\
\hline Aneides hardii & RAC042 & NM: Lin & MF946488 & MF946513 & MF946538 & This study \\
\hline Aneides hardii & RAC054 & NM: Lin & MF946489 & MF946514 & MF946539 & This study \\
\hline Aneides lugubris & MVZ230722 & CA: San Diego & MF946490 & MF946515 & MF946540 & This study \\
\hline Aneides lugubris & MVZ249828 & CA: Mariposa & MF946491 & MF946516 & MF946541 & This study \\
\hline Aneides lugubris & RAC060 & CA: Santa Clara & MF946492 & MF946517 & MF946542 & This study \\
\hline Aneides lugubris & RAC081 & CA: Mendocino & MF946493 & MF946518 & MF946543 & This study \\
\hline Aneides vagrans & HBS26688 & CA: Mendocino & MF946494 & MF946519 & MF946544 & This study \\
\hline Aneides vagrans & MVZ219886 & CA: Del Norte & MF946495 & MF946520 & MF946545 & This study \\
\hline Aneides vagrans & MVZ220991 & CA: Humboldt & MF946496 & MF946521 & MF946546 & This study \\
\hline Aneides vagrans & RAC073 & CA: Humboldt & MF946497 & MF946522 & MF946547 & This study \\
\hline Ensatina eschscholtzii & MVZ236171 & CA: San Luis Obispo & EU275862 & EU275816 & EU275785 & Vieites et al. [38] \\
\hline Desmognathus fuscus & MVZ224931 & MA: Franklin & EU275858 & EU275812 & EU275781 & Vieites et al. [38] \\
\hline Hydromantes brunus & MVZ238576 & CA: Mariposa & EU275871 & EU275825 & EU275790 & Vieites et al. [38] \\
\hline Plethodon elengatus & MVZ220003 & CA: Del Norte & EU275882 & EU275836 & AY650120 & Wiens et al. [58]; Vieites et al. [38] \\
\hline
\end{tabular}

"heated" and one "cold" Markov chain for 10 million generations with every 1000th sample retained. A rate multiplier was used to allow substitution rates to vary among partitions, and default priors were applied to all model parameters. We assessed convergence of the MCMC using several diagnostics. We viewed trace plots of tree - $\operatorname{lnL}$ values and other parameters in Tracer v.1.5 [43]. Trees sampled prior to stationarity (i.e. the first 1000 tree samples) were considered as burn in and were discarded. To determine whether the two independent runs converged on similar results, we examined the split standard deviation for $-\ln L$ tree values among chains; values $<0.01$ were taken to indicate convergence. We also used the program Are We There Yet? (AWTY) [44] to compare changes in the posterior probabilities of split frequencies across the independent runs.

Partitioned Maximum Likelihood analyses were conducted using RAxML v.7.2.5 [45] under the GTR + $\Gamma$ model of nucleotide substitution for all data partitions identified in the Bayesian analysis. Support values for the inferred relationships were obtained from 1000 nonparametric bootstrap pseudoreplicates.

\section{Mitochondrial genome sequencing and assembly}

We obtained cellular DNA shotgun sequence data, which includes both nuclear and mitochondrial DNA, using the 
Table 2 Individuals used in this study and their voucher numbers, total number of Illumina MiSeq reads, total number of base pairs, total number of contigs used in mitochondrial genome assembly, and sequence length (kb) of mitochondrial genome annotation

\begin{tabular}{|c|c|c|c|c|c|}
\hline Species & Voucher & Number of reads & Total base pairs & $\begin{array}{l}\text { Mitochondrial } \\
\text { contigs }\end{array}$ & $\begin{array}{l}\text { Mitochondrial } \\
\text { genome annotation (kb) }\end{array}$ \\
\hline A. aeneus & DH77584 & $1,648,165$ & $510,564,223$ & 1 & 16.8 \\
\hline A. ferreus & RCT545 & $1,181,785$ & $365,727,056$ & 5 & 16.4 \\
\hline A. flavipunctatus & MVZ219977 & $1,165,899$ & $349,989,009$ & 5 & 17.1 \\
\hline A. flavipunctatus & RLM172 & $1,044,399$ & $308,615,225$ & 4 & 17.5 \\
\hline A. flavipunctatus & AY728214 & - & - & 1 & 20.2 \\
\hline A. hardii & RAC25 & 931,319 & $284,267,433$ & 6 & 18.7 \\
\hline A. hardii & RAC42 & $1,708,754$ & $507,960,897$ & 5 & 17.9 \\
\hline A. hardii & RAC54 & $1,280,531$ & $378,635,736$ & 1 & 17.0 \\
\hline A. hardii & AY728226 & - & - & 1 & 22.2 \\
\hline A. lugubris & MVZ249828 & 728,185 & $199,882,489$ & 7 & 17.0 \\
\hline A. vagrans & MVZ220991 & 832,332 & $252,211,876$ & 5 & 16.5 \\
\hline
\end{tabular}

Illumina MiSeq sequencing platform for a total of eight individuals representing all six species of Aneides, with additional sampling within $A$. hardii and A. flavipunctatus (Table 2). Illumina sequencing libraries were produced for each sample using the IntegenX PrepX-DNA 24 library prep kit (IntegenX). Libraries were pooled equimolar for sequencing, allocating $1 / 3$ of a $2 \times 250$ cycle MiSeq run (Illumina) for all samples. Library preparation and sequencing were performed by the University of Idaho Institute for Bioinformatics and Evolutionary Studies (IBEST) Genomics Resources Core facility. All sequences are deposited in the GenBank SRA (PRJNA407969). We screened all shotgun reads to eliminate sequencing adapters, identify and remove contaminants, and trim reads based on quality scores using the bioinformatics pipeline "SeqyClean.py" provided by the IBEST Computational Resources Core. We assembled the remaining shotgun reads into contigs using Newbler v.2.6. We also included published shotgun sequence data for an additional sample of $A$. flavipunctatus [46], resulting in shotgun datasets for nine total individuals.

For each of the nine shotgun sequence datasets, we identified contigs of mitochondrial sequences using tBLASTx; the mitochondrial genome reference sequences of $A$. hardii (AY728226) and A. flavipunctatus (AY728214) were used as queries to BLAST against the assembled contigs with an e-value cutoff of 1e-5. Depending on the focal individual, complete (or nearly complete) mitochondrial genomes were represented by one to eight contigs. Several genome assemblies included gaps across regions of low/non-existent sequencing coverage. To close these gaps, as well as verify the assembly of multiple contigs, we developed genome-specific primers for PCR amplification and Sanger sequencing. PCR amplification and Sanger sequencing were performed as described above for the nuclear genes used in the phylogenetic analysis.

\section{Mitochondrial gene order identification}

We annotated mitochondrial genome sequences for each individual using Geneious R7. rRNAs and tRNAs were identified based on sequence similarity with published genomes. All 13 protein-coding sequences were verified by eye for appropriate vertebrate mitochondrial open reading frames and stop codons. For each sample, we determined mitochondrial gene order based on our genome annotations and alignment to reference mitochondrial genomes. We also identified pseudogenes based on sequence similarity to inferred functional gene copies as well as position relative to annotated gene sequences.

\section{Gene loss analysis}

Within $A$. hardii, individuals have different mitochondrial genome haplotypes; one haplotype has additional duplicate genes not present in the other. We thus focused on A. hardii to study random gene loss "in action." To this end, we measured (1) substitutions within duplicate gene sequences and their effects on open reading frame, if applicable, and (2) deletions within duplicate sequences. To perform these measures, we created pairwise alignments of pseudogene sequences and their intact paralogs from within the same genome using ClustalW; the intact paralog serves as a proxy for the sequence immediately following duplication. We scaled deletions of duplicate sequences by substitutions, as has been done in other studies, because the limited salamander fossil record precludes precise estimates of absolute rates; salamander divergence date estimates have large 95\% confidence intervals [28, 38, 47-49]. More specifically, we estimated the average number of mitochondrial nucleotide 
substitutions per site $\left(D_{X Y}\right)$ between the two different $A$. hardii gene orders. We generated multiple sequence alignments including two individuals representing each gene order for each of the protein-coding genes using translational ClustalW alignment [50] and verified open reading frames and stop codons by eye (see Additional file 1). We then estimated $D_{X Y}$ for a concatenated dataset of all 13 mitochondrial protein-coding genes using DnaSP v 5.10.1 [51], which uses the Jukes and Cantor's model of nucleotide substitution to estimate genetic divergence between populations and is likely sufficient for such shallow divergences.

\section{Results and discussion}

\section{Aneides phylogeny estimated from nuclear genes}

Despite recent work on salamander phylogenies [38, 39, 52, 53], the phylogenetic relationships within Aneides were previously unknown. From our analysis of $2046 \mathrm{bp}$ from three nuclear genes (BDNF, POMC, and RAG1), we estimated a resolved phylogeny for the genus that includes all six species represented by several divergent lineages (Fig. 1). Our results show A. aeneus to be sister to the rest of the clade; $A$. aeneus is found in eastern North America, while the remaining five species are only found in western North America. The two Pacific Northwest species - A. ferreus and A. vagrans - are sister taxa, which together form the clade sister to A. flavipunctatus. Aneides flavipunctatus $+A$. ferreus $+A$. vagrans is sister to $A$. lugubris; these four species are found in the coastal regions of Western North America. Aneides hardii, which is restricted to a few isolated mountaintops in southern New Mexico, is sister to the coastal clade. Our results are consistent with the relationships suggested by other studies that included some species of Aneides [32, 38, 39, 53].

\section{Mitochondrial gene order}

We identified mitochondrial gene orders for nine Aneides samples using shotgun sequence data. The total amount of sequence and average read length varied among samples, but overall, the datasets represent $\sim 0.5-1.5 \%$ of the nuclear genomes at $1 \times$ coverage (Table 2 ). We produced nearly complete mitochondrial genome sequences from a single contig for two samples. For each of the remaining samples, we produced four to eight contigs that represent large fractions of the mitochondrial genome. We eliminated several assembly gaps using PCR; however, several regions of the genome remain absent from our assemblies. Specifically, the control region and the region between

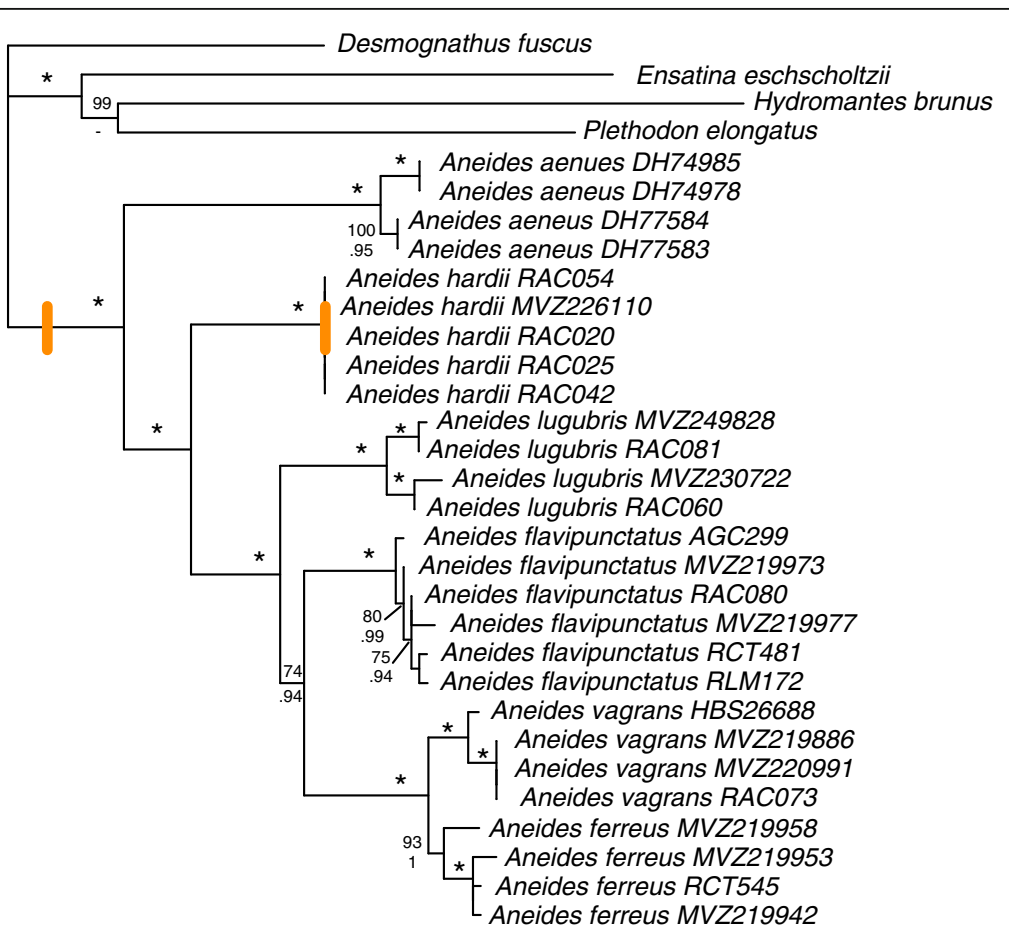

Duplication \& Rearrangement

0.01 substitutions/site

Fig. 1 Nuclear phylogeny of Aneides with nodes labeled with Maximum Likelihood bootstrap support (MLBP) above and Bayesian posterior probabilities (BPP) below. Both analyses resulted in congruent tree topologies, depicted above, with highly supported nodes (MLBP >99 and BPP > 0.99) denoted with an asterisk, while weakly supported nodes (MLBP $<70$ or BPP $<0.70$ ) are not labeled. Aneides hardii branch lengths are all $<0.00001$. Orange bars indicate inferred mitochondrial gene duplication and rearrangement events 
CYTB and ND6 were difficult to assemble, likely reflecting the highly repetitive nature of these two regions [19]. Despite these gaps, our contigs include all 37 mitochondrial genes for each individual; thus, we were able to infer gene order from all nine individuals with confidence. We combined these data with previously published genomes from two additional Aneides individuals (one $A$. hardii and one A. flavipunctatus) for a total of 11 focal Aneides mitochondrial genome sequences (Table 2).

We found evidence of gene rearrangement, relative to the typical vertebrate mitochondrial gene order, in all 11 mitochondrial genome sequences. The mitochondrial gene order in A. aeneus, A. lugubris, A. vagrans, A. ferreus, all sampled lineages of $A$. flavipunctatus, and a subset of the sampled lineages of $A$. hardii includes a gene order exchange between ND6 and CYTB as well as the accumulation of repetitive, non-coding DNA in this region (Fig. 2a). This pattern is consistent with (1) an initial duplication of ND6, trn-E, CYTB, trn-T, an intergenic spacer, and trn-P, followed by (2) complete excision of one copy of ND6+trn-E and decay of the remaining sequences to either recognizable pseudogenes (i.e. $\psi$ trn-P) or unrecognizable tandem repeats [19]. Based on these results, we infer that this gene rearrangement is a synapomorphy for Aneides that occurred at the base of the clade (Fig. 1). Divergence date estimates for the split between Aneides and its sister taxon (i.e. Desmognathus + Phaeognathus) range from 36 to 69 mya, providing maximum and minimum dates for this duplication [38]. Thus, non-coding DNA resulting from the gene duplication at the base of Aneides has persisted in the genome of the descendant lineages for tens of millions of years.
Gene order is polymorphic within A. hardii

Of the four new $A$. hardii mitochondrial genomes we sequenced for this study, two individuals have the gene order we show here to be ancestral for Aneides (Fig. 2a). The other two individuals have the gene order previously reported for this species that reflects two duplication events [19] (Fig. 2b). Thus, within A. hardii, we identified a recent gene rearrangement where duplicates and intermediate stages of paralog loss are polymorphic within the species. Although our sampling found the gene orders in geographically isolated populations, our sample size is too small to infer that the populations are fixed for the difference in gene order.

\section{Patterns of gene loss following duplication in A. hardii}

Since the duplication event occurred within the $A$. hardii lineage, paralogous sequences within $A$. hardii genomes have begun to diverge from one another, accumulating point substitutions, deletions, and, to a lesser extent, insertions. Here, we summarize patterns of divergence between duplicates for different types of sequences.

\section{Protein-coding genes}

All of ND6 and at least some portion of ND1 were included in the $A$. hardii-specific duplication (Fig. 2a). The functional copy of ND6 in $A$. hardii is 519 bp long. In contrast, the putative pseudogene copy of ND6 we identified in RAC25 is 521 bp long, reflecting one insertion of a total length of two bp. We were unable to assemble this region of the genome in RAC20. Sequence identity between ND6 and 4 ND6 is 97\%. $\psi$ ND6 differs from the functional copy by ten point substitutions and two insertions, which result in several amino acid replacements to

\section{a}

Aneides-specific gene order
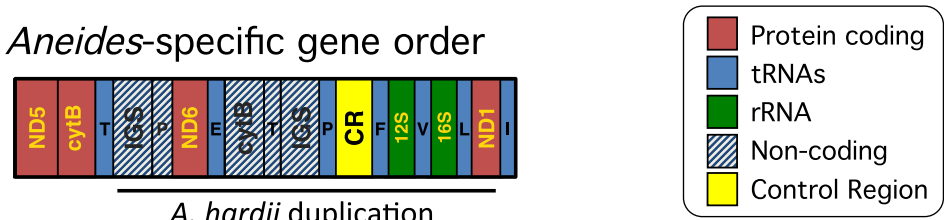

A. hardii duplication

Control Region

b

Inferred intermediate gene order

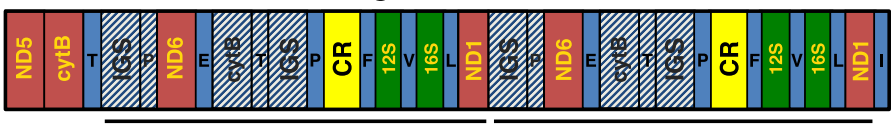

A. hardii-specific gene order

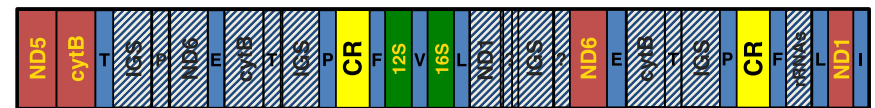

Fig. 2 Mitochondrial gene rearrangements in Aneides hardii. a Aneides-specific mitochondrial gene order, which is ancestral for A. hardii. The region inferred to have been duplicated in the $A$. hardii-specific duplication event is underlined. $\mathbf{b}$ Inferred intermediate gene order after A. hardii-specific duplication event and the current novel gene order within $A$. hardii 
stop codons. The longest predicted ORF within $\psi$ ND6 is 139 amino acids long.

The functional copy of ND1 in A. hardii is $960 \mathrm{bp}$. $\psi \mathrm{ND} 1$ is a 676 and 685 bp fragment that shares $91.7 \%$ and $94.6 \%$ sequence identity with the first $682 \mathrm{bp}$ of the functional ND1 in RAC25 and RAC20, respectively. Specifically, $\psi$ ND1 differs from the functional copy in RAC20 by 30 point substitutions, a 2 bp deletion, and two insertions (5 bp total), which result in several amino acid replacements to stop codons. Similarly, $\psi$ ND1 differs from the functional copy in RAC25 by 42 point substitutions, four deletions (11 bp total), and two insertions (5 bp total), resulting in several amino acid replacements to stop codons. The longest predicted ORF within $\psi$ ND1 is 105 amino acids long in RAC25 and 205 amino acids in RAC20. The $\psi$ ND1 sequences are $94.7 \%$ identical between the two individuals. Because we are unable to confirm the total amount of ND1 that was included in the initial duplication, we are unable to determine the total amount of deleted sequence in $\psi \mathrm{ND} 1$. If ND1 were only partially duplicated, it would have become a pseudogene immediately because of its shortened length.

\section{rRNA genes}

Both ribosomal RNA genes were included in the A. hardiispecific duplication. Duplicate copies of both rRNAs, as well as the intervening tRNA- $\mathrm{V}$, have been almost completely deleted from genomes with the $A$. hardii-specific duplication. The functional copies of these three genes together total $2520 \mathrm{bp}$ of contiguous sequence; however, only a 111-bp-long segment of unrecognizable sequence remains of the duplicates.

\section{tRNA genes}

tRNA-E, $-\mathrm{P},-\mathrm{F},-\mathrm{V}$, and $-\mathrm{L}$ were included in the $A$. hardii-specific duplication. With the exception of one tRNA, we were able to identify all $A$. hardii-specific duplicated tRNA gene copies (except tRNA-V, located between the two rRNAs), which appear to largely remain functional based on intact stem-loop structures and the anticodon sequences. Both individuals with the $A$. hardiispecific duplication retain identical copies of tRNA-P and likely tRNA-E as well (although we were unable to identify a second copy in RAC20). tRNA-L paralog sequences differed by two single-basepair substitutions (one shared, one unique) in both individuals and share $97.7 \%$ sequence identity, even though each tRNA sequence is unique. For individuals with the $A$. hardii-specific duplication, tRNA-F sequences upstream of the intact rRNA genes are identical, while tRNA-F sequences adjacent to the pseudogenized rRNA genes are more variable and likely nonfunctional. The latter tRNA-F differs from the former by three bases (in RAC20) and five bases (in RAC25), with only two differences being shared by both individuals. The relatively short length of tRNA genes ( $70 \mathrm{bp}$ ) limits the likelihood of accumulating substitutions and indels within these genes, which would allow for the conservation and functional persistence of tRNAs over other duplicated genes of greater length.

\section{Non-coding sequences}

Two regions of non-coding, repetitive DNA remain in all Aneides mitochondrial genomes from the ancestral duplication at the base of the clade. These regions are predicted to be the remnants of (1) a copy of CYTB + tRNA-T + IGS and (2) a copy of IGS + tRNA-P. Both were included in the $A$. hardii-specific duplication, and at least a portion of both sequences is retained in duplicate following the $A$. hardii duplication. We were unable to fully resolve these regions of the genome because they are repetitive and could not be assembled with confidence. Average sequencing read depth of these regions is at least double that of the rest of the genome, suggesting that these regions occur at least twice as often.

Taken together, our results show that, following the $A$. hardii-specific duplication event, different duplicate sequences have had dramatically different fates. Ribosomal gene copies have been almost completely deleted from the genome, and the sequence remaining bears no identity to the functional rRNA copies. In contrast, duplicate proteincoding sequences have not been deleted; specifically, $\psi$ ND6 is now longer than the functional paralog, and $\psi \mathrm{ND} 1$ does not show evidence of substantial deletion. Both duplicated protein-coding sequences have decayed to pseudogenes and now encode short, presumably non-functional ORFs. Duplicate tRNAs (except trn-V) also show no evidence of deletion; tRNA-E, -P, and -L remain apparently functional, retaining $98-100 \%$ sequence similarity between the two paralogs.

\section{Loss of duplicate sequences in A. hardii occurred over a shallow evolutionary timescale}

We measured the number of substitutions between the $A$. hardii mitochondrial genomes that (1) retained the ancestral Aneides gene order, and (2) underwent the $A$. hardii-specific duplication. The average number of nucleotide substitutions per site between the two different mitochondrial gene orders found within $A$. hardii was low $\left(D_{X Y}=0.024\right)$. This result is congruent with our nuclear gene phylogenetic analysis, which shows very low genetic divergence among $A$. hardii individuals (Fig. 1). Thus, all of the duplicate gene sequence decay/loss we identify in the $A$. hardii-specific duplication has occurred in the time taken to accumulate only $2.4 \%$ sequence divergence from the ancestral Aneides mitochondrial genomes. 


\section{What evolutionary forces may have shaped duplicate gene loss in $A$. hardii?}

We have demonstrated that two mitochondrial gene duplication events occurred during the history of the genus Aneides - one tens of millions of years ago, and another much more recently within a single species. We have shown that different duplicate sequences have had very different fates: ribosomal gene copies have been almost completely deleted from rearranged Aneides hardii genomes, whereas non-coding sequence from the duplication event that occurred at the base of the clade persists in several species of Aneides. What do these patterns of paralogous sequence evolution suggest about the evolutionary forces that shape duplicate gene loss in mitochondria?

The evolutionary history of the mitochondrial genome, from its inception as a bacterial endosymbiont to its emergence as an essential organellar genome, includes massive genomic reduction [54-56]. Consistent with this overall trend, it has been hypothesized that selection for efficiency of mitochondrial replication and transcription would favor the elimination of extraneous sequence. Additionally, the accumulation of noncoding DNA can pose a mutational hazard [3-5, 57]. In Aneides, we do not see evidence that deletions of extraneous sequence are frequent targets of strong positive selection. Rather, we see (1) non-coding sequences persisting/expanding in length in all species of Aneides that originated from a duplication event tens of millions of years ago, (2) non-coding sequences (i.e. repeat sequences and pseudo-protein-coding genes) persisting in the $A$. hardii genomes that underwent a duplication event much more recently, and (3) fulllength duplicate tRNAs persisting in $A$. hardii genomes, all of which suggest that these genomes do not rapidly purge duplicated regions. These patterns have at least two possible (somewhat overlapping) interpretations. First, it is possible that duplicate sequences do not strongly negatively impact mitochondrial function through their effects on rates of duplication and transcription or their mutational hazard. Accordingly, insertions and deletions in these sequences reflect the underlying mutational spectrum, and large deletions are relatively rare. Second, it is possible that large deletions may have enough of an impact on duplication/transcription/harmful mutation rate to be targets of positive selection, but that such large deletions are sufficiently rare, relative to substitutions and smaller insertions and deletions, that duplicate sequences persist in Aneides genomes, despite some negative fitness consequences.

In contrast to the persistence of duplicate tRNAs and pseudogenized protein-coding genes, as well as the persistence/expansion of repetitive sequence, duplicate ribosomal RNA genes have been almost completely deleted since the recent $A$. hardii duplication event. This suggests that selection may have favored deletion of these sequences. Although the presence of additional identical rRNA gene copies in the mitochondrial genome may be unlikely to have deleterious fitness consequences, the presence of pseudo-rRNA genes that compromise protein translation could negatively impact fitness. Thus, we hypothesize that (1) duplicate rRNA genes sustained mutations that compromised translational capacity, and (2) deletions eliminating such sequences were then favored by selection. With our data, however, we cannot exclude the alternate possibilities that a large deletion eliminating the duplicate rRNA genes was fixed by genetic drift or by selection acting on duplication/transcription. Characterizing the evolutionary fate of other recent rRNA gene duplications would aid in discriminating among these hypotheses.

\section{Conclusions}

Although metazoan mitochondrial genomes have conserved gene content, gene order can vary across taxa, reflecting partial genome duplication and subsequent paralog loss. We identified two duplication events in the history of the salamander genus Aneides: one that occurred tens of millions of years ago at the base of the clade, and the other that occurred recently within a single species (A. hardii). Our results suggest that some extraneous sequences have remained in Aneides mitochondrial genomes for tens of millions of years, while duplicate rRNA genes have been eliminated over much shorter timescales. Together with previous work showing that (1) increased metabolic demand is not correlated with mitochondrial genome streamlining in amphibians [21], and (2) genome expansion does not place genes in more vulnerable positions along a strong mutation gradient in salamanders [23], our results suggest that overall DNA loss is not likely to be a strong target of selection in salamander mitochondrial genomes. This parallels the pattern seen in salamander nuclear genomes, which also show slow rates of DNA loss [47]. Further studies identifying recent rRNA duplications in other taxa are required to test whether additional rRNA genes are functionally disadvantageous and targeted by purifying selection.

\section{Additional file}

Additional file 1: ClustalW-created multiple sequence alignments for each of the mitochondrial protein-coding genes including two individuals representing each $A$. hardii gene order. (TXT $45 \mathrm{~kb}$ )

\footnotetext{
Acknowledgements

This work was supported by the National Science Foundation [NSF-DEB 1021489 to RLM and NSF-DEB 1210900 to RAC and RLM] and by Colorado State University. M. Settles provided much helpful discussion of data collection and analysis. We thank members of RAC's thesis committee (C.T. Webb, W. C. Funk, and J. McKay) for helpful discussion and comments on the manuscript.
} 


\section{Funding}

This work was supported by the National Science Foundation [NSF-DEB 1021489 to RLM and NSF-DEB 1210900 to RAC and RLM] and by Colorado State University.

\section{Availability of data and materials}

The datasets supporting the conclusions of this article are available in the GenBank repository (https://www.ncbi.nlm.nih.gov/genbank/), and we have provided a table listing corresponding accession numbers. Datasets supporting the conclusions of this article are also included within the article and its additional file.

\section{Authors' contributions}

RAC and RLM contributed to the conception and design of the study, RAC collected and analyzed the data, RAC and RLM wrote the manuscript. Both authors read and approved the final manuscript.

\section{Ethics approval}

All work was completed in compliance with Colorado State University's Institutional Animal Care and Use Committee (Protocol 08-184A-02).

\section{Consent for publication}

Not applicable

\section{Competing interests}

The authors declare that they have no competing interests.

\section{Publisher's Note}

Springer Nature remains neutral with regard to jurisdictional claims in published maps and institutional affiliations.

\section{Received: 24 December 2016 Accepted: 29 November 2017}

\section{Published online: 28 December 2017}

\section{References}

1. Boore JL. Animal mitochondrial genomes. Nucleic Acids Res. 1999;27(8): 1767-80.

2. Scheffler IE. Mitochondria. 2nd ed. New Jersey: Wiley; 2008.

3. Allen JF, Puthiyaveetil S, Strom J, Allen CA. Energy transduction anchors genes in organelles. BioEssays. 2005;27:426-35.

4. Wright AF, Murphy MP, Turnbull DM. Do organellar genomes function as long-term redox damage sensors? Trends Genet. 2009;25(6):253-61.

5. Daley DO, Whelan J. Why genes persist in organelle genomes. Gen Biol. 2005;6:110.

6. Boore $J$, Brown WM. Big trees from little genomes: mitochondrial gene order as a phylogenetic tool. Curr Opin Genet Dev. 1998;8(6):668-74.

7. Saccone C, De Giorgi C, Gissi C, Pesole G, Reyes A. Evolutionary genomics in Metazoa: the mitochondrial DNA as a model system. Gene. 1999;238(1):195209.

8. Xu W, Jameson D, Tang B, Higgs PG. The relationship between the rate of molecular evolution and the rate of genome rearrangement in animal mitochondrial genomes. J Mol Evol. 2006;63(3):375-92.

9. Xia $Y$, Zheng $Y$, Miura I, Wong PB, Murphy RW, Zeng $X$. The evolution of mitochondrial genomes in modern frogs (Neobatrachia): nonadaptive evolution of mitochondrial genome reorganization. BMC Genomics. 2014;15:691

10. Aguileta G, de Vienne DM, Ross ON, Hood ME, Giraud T, Petit E, Gabaldon T. High variability of mitochondrial gene order among fungi. Gen Biol Evol. 2014;6(2):451-65.

11. Satoh T, Sato Y, Masuyama N, Miya M, Nishida M. Transfer RNA gene arrangement and codon usage in vertebrate mitochondrial genomes: a new insight into gene order conservation. BMC Genomics. 2010;11(1):479.

12. Boore JL. The duplication/random loss model for gene rearrangement exemplified by mitochondrial genomes of deuterostome animals. Comp Genom. 2000;1:133-47.

13. Moritz C, Dowling TE, Brown WM. Evolution of animal mitochondrial DNA: relevance for population biology and systematics. Ann Rev Ecol Evol Syst. 1987:18:269-92.

14. Fujita MK, Boore JL, Moritz C. Multiple origins and rapid evolution of duplicated mitochondrial genes in parthenogenetic geckos (Heteronotia binoei; Squamata, Gekkonidae). Mol Biol Evol. 2007;24(12):2775-86.
15. Mortiz C, Brown WM. Tandem duplications in animal mitochondrial DNAs: variation in incidence and gene content among lizards. Proc Nat Acad Sci. 1987;84(20):5

16. San Mauro D, Gower DJ, Zardoya R, Wilkinson M. A hotspot of gene order rearrangement by tandem duplication and random loss in the vertebrate mitochondrial genome. Mol Biol Evol. 2006;23(1):227-34.

17. Shao R, Dowton M, Murrell A, Barker SC. Rates of gene rearrangement and nucleotide substitution are correlated in the mitochondrial genomes of insects. Mol Biol Evol. 2003;20(10):1612-9.

18. Kumazawa Y, Miura S, Yamada C, Hashiguchi Y. Gene rearrangements in gekkonid mitochondrial genomes with shuffling, loss, and reassignment of tRNA genes. BMC Genomics. 2014;15:930.

19. Mueller RL, Boore JL. Molecular mechanisms of extensive mitochondrial gene rearrangement in plethodontid salamanders. Mol Biol Evol. 2005; 22(10):2104-12.

20. Selosse MA, Albert B, Godelle B. Reducing the genome size of organelles favours gene transfer to the nucleus. Trends Ecol Evol. 2001;16(3):135-41.

21. Chong RA, Mueller RL. Low metabolic rates in salamanders are correlated with weak selective constraints on mitochondrial genes. Evolution. 2013; 67(3):894-9.

22. Lynch M, Bobay LM, Catania F, Gout JF, Rho M. The repatterning of eukaryotic genomes by random genetic drift. Ann Rev Genom Hum Genet. 2011;12:347-66.

23. Chong RA, Mueller RL. Evolution along the mutation gradient in the dynamic mitochondrial genome of salamanders. Gen Biol Evol. 2013;5(9): 1652-60.

24. Schuster W, Brennicke A. RNA editing makes mistakes in plant mitochondria: editing loses sense in transcripts of a rps19 pseudogene and in creating stop codons in coxl and rps3 mRNAs of Qenothera. Nuc Ac Res. 1991:19(24):6923-8.

25. Papa S, Skulachev VP. Reactive oxygen species, mitochondria, apoptosis and aging. Mol Cell Biochem. 1997;174:305-19.

26. Rossi CS, Lehninger AL. Stoichiometry of respiratory stimulation, accumulation of $\mathrm{Ca}++$ and phosphate, and oxidative phosphorylation in rat liver mitochondria. J Biol Chem. 1964;239(11):3971-80.

27. Haring E, Kruckenhauser L, Gamauf A, Riesing MJ, Pinsker W. The complete sequence of the mitochondrial genome of Buteo buteo (Aves, Accipitridae) indicates an early split in the phylogeny of raptors. Mol Biol Evol. 2001;18(10):13.

28. McKnight ML, Shaffer HB. Large, rapidly evolving intergenic spacers in the mitochondrial DNA of the salamander family Ambystomatidae (Amphibia: Caudata). Mol Biol Evol. 1997;14(11):1167-76.

29. Raboin MJ, Timko AF, Howe DK, Felix M-A, Denver DR. Evolution of Caenorhabditis mitochondrial genome pseudogenes and Caenorhabditis briggsae natural isolates. Mol Biol Evol. 2010;27(5):1987-096.

30. Xia Y, Zheng Y, Murphy RW, Zeng X. Intraspecific rearrangement of mitochondrial genome suggests the prevalence of the tandem duplicationrandom loss (TDLR) mechanism in Quasipaa boulengeri. BMC Genomics. 2016;17(1):965

31. AmphibiaWeb. http://amphibiaweb.org. University of California, Berkeley, CA; 2017

32. Mueller RL, Macey JR, Jaekel M, Wake DB, Boore JL. Morphologica homoplasy, life history evolution, and historical biogeography of plethodontid salamanders inferred from complete mitochondrial genomes. Proc Natl Acad Sci U S A. 2004;101(38):13820-5.

33. Zhang $P$, Chen $Y-Q$, Liu Y-F, Zhou H, L-H Q. The complete mitochondrial genome of the Chinese giant salamander, Andrias davidianus (Amphibia: Caudata). Gene. 2003:311:93-8.

34. Zhang P, Chen Y-Q, Zhou H, Wang X-L, L-H Q. The complete mitochondrial genome of a relic salamander, Ranodon sibiricus (Amphibia: Caudata) and implications for amphibian phylogeny. Mol Phylogen Evol. 2003;28(3):620-6.

35. Rissler $\sqcup$, Apodaca JJ. Adding more ecology into species delimitation: ecological niche models and phylogeography help define cryptic species in the black salamander (Aneides flavipunctatus). Syst Biol. 2007;56(6):924-42.

36. Pope $\mathrm{MH}$, Highton R. Geographic genetic variation in the Sacramento Mountain salamander, Aneides hardii. J Herp. 1980;14(4):343-6.

37. Reilly SB, Wake DB. Cryptic diversity and biogeographical patterns within the black salamander (Aneides flavipunctatus) complex. J Biogeogr. 2015; 42(2):280-91.

38. Vieites DR, Min MS, Wake DB. Rapid diversification and dispersal during periods of global warming by plethodontid salamanders. Proc Natl Acad Sci U S A. 2007;104(50):19903-7. 
39. Vieites DR, Roman SN, Wake MH, Wake DB. A multigenic perspective on phylogenetic relationships in the largest family of salamanders, the Plethodontidae. Mol Phylogenet Evol. 2011;59(3):623-35.

40. Edgar RC. MUSCLE: multiple sequence alignment with high accuracy and high throughput. Nuc Acid Res. 2004;32(5):1792-7.

41. Lanfear R, Calcott B, Ho SY, Guindon S. Partitionfinder: combined selection of partitioning schemes and substitution models for phylogenetic analyses. Mol Biol Evol. 2012;29(6):1695-701.

42. Ronquist F, Teslenko M, van der Mark P, Ayres DL, Darling A, Hohna S, Larget B, Liu L, Suchard MA, Huelsenbeck JP. MrBayes 3.2: efficient Bayesian phylogenetic inference and model choice across a large model space. Syst Biol. 2012;61(3):539-42.

43. Rambaut A, Drummond A: Tracer v1.5. http://beastbioedacuk/Tracer 2007.

44. Nylander JAA, Wilgenbusch JC, Warren DL, Swofford DL. AWTY (are we there yet?): a system for graphical exploration of MCMC convergence in Bayesian phylogenetics. Bioinformatics. 2008;24(4):3.

45. Stamatakis A. RAxML-VI-HPC: maximum likelihood-based phylogenetic analyses with thousands of taxa and mixed models. Bioinformatics. 2006; 22(21):2688-90.

46. Sun C, Shepard DB, Chong RA, Lopez Arriaza J, Hall K, Castoe TA, Feschotte C, Pollock DD, Mueller RL. LTR retrotransposons contribute to genomic gigantism in plethodontid salamanders. Gen Biol Evol. 2012;4(2):168-83.

47. Sun C, Lopez Arriaza JR, Mueller RL. Slow DNA loss in the gigantic genomes of salamanders. Gen Biol Evol. 2012;4(12):1340-8.

48. Petrov DA. Evidence for DNA loss as a determinant of genome size. Science. 2000;287(5455):1060-2.

49. Mueller RL. Evolutionary rates, divergence dates, and the performance of mitochondrial genes in Bayesian phylogenetic analysis. Syst Biol. 2006;55(2): 289-300.

50. Larkin MA, Blackshields G, Brown NP, Chenna R, McGettigan PA, McWilliam $H$, Valentin F, Wallace IM, Wilm A, Lopez R, et al. Clustal W and Clustal X version 2.0. Bioinformatics. 2007;23(21):2947-8.

51. Librado P, Rozas J. DnaSP v5: a software for comprehensive analysis of DNA polymorphism data. Bioinformatics. 2009;25(11):1451-2.

52. Kalinowski ST, Taper ML, Marshall TC. Revising how the computer program CERVUS accommodates genotyping error increases success in paternity assignment. Mol Ecol. 2007;16(5):1099-106.

53. Pyron RA, Wiens JJ. A large-scale phylogeny of Amphibia including over 2800 species, and a revised classification of extant frogs, salamanders, and caecilians. Mol Phylogen Evol. 2011;61(2):543-83.

54. Andersson SG, Karlberg O, Canback B, Kurland CG. On the origin of mitochondria: a genomics perspective. Phil Trans Roy Soc London Ser B. 2003;358(1429):165-77. discussion 177-169

55. Andersson SG, Zomorodipour A, Andersson JO, Sicheritz-Ponten T, Alsmark UC, Podowski RM, Naslund AK, Eriksson AS, Winkler HH, Kurland CG. The genome sequence of Rickettsia prowazekii and the origin of mitochondria. Nature. 1998;396(6707):133-40.

56. Burger G, Gray MW, Franz Lang B. Mitochondrial genomes: anything goes. Trends Genet. 2003;19(12):709-16.

57. Lynch M, Koskella B, Schaack S. Mutation pressure and the evolution of organelle genomic architecture. Science. 2006;311(5768):1727-30.

58. Wiens JJ, Bonett RM, Chippindale PT. Ontogeny discombobulates phylogeny: Paedomorphosis and higher-level salamander relationships. Syst Biol. 2005; 54(1):91-110

\section{Submit your next manuscript to BioMed Central and we will help you at every step:}

- We accept pre-submission inquiries

- Our selector tool helps you to find the most relevant journal

- We provide round the clock customer support

- Convenient online submission

- Thorough peer review

- Inclusion in PubMed and all major indexing services

- Maximum visibility for your research

Submit your manuscript at www.biomedcentral.com/submit

) Biomed Central 